\title{
ESTUDO ARQUEOMÉTRICO DE CERÂMICAS DO SÍTIO GUARÁ, GOIÁS, BRASIL
}

\section{Claudia V. Schayer Sabino*, Otaviano F. N. Neves e Milton B. Franco}

Centro de Desenvolvimento da Tecnologia Nuclear, Comissão Nacional de Energia Nuclear, CP 941, 30123-970 Belo Horizonte - MG Irmhild Wust

Departamento de Ciências Sociais, Museu Antropológico, Universidade de Goiás, Alameda das Rosas, s/n, 74610-130 Goiás - GO Andre P. Prous

Museu de História Natural, Universidade Federal de Minas Gerais, Rua Gustavo da Silveira, 1035, 30080-010 Belo Horizonte - MG

Recebido em 5/2/01; aceito em 14/8/01

\begin{abstract}
ARCHAEOMETRIC STUDY OF GUARÁ, GOIÁS, BRAZIL CERAMICS. There is no evidence of urban civilization in Brazilian prehistory; most inhabitants lived in tribal groupings, probably with regional economic integration among several independent tribes. There is little evidence of seasonal migrations between the coast and the inland of southern Brazil. Some specialized horticulturists competed among themselves but other groups lived more isolated and probably peacefully, in the upper interfluvial regions. Chemical analysis of artifacts is a means of documenting traffic in particular materials and intraregional production and distribution, development of craft specialization and typological refinement among other issues.

In this study we tested some possibilities in two different cultural contexts using the parametric $\mathrm{k}_{0}$ neutron activation analysis technique, which allowed the determination of elements: Al, As, Au, Ce, Cl, Co, Cr, Cs, Cu, Fe, Ga, K, La, Na, Rb, Sc, Ta, Ti, V and Zn.
\end{abstract}

Keywords: archaeometry; neutron activation analysis; archaeological pottery.

\section{INTRODUÇÃO}

O uso da química analítica em estudos arqueológicos remonta a 1700. Uma das principais finalidades de tais estudos era verificar o tráfego de peças de cerâmicas e a interação entre povos. Hoje, as técnicas são utilizadas não apenas com o objetivo inicial, mas também no estudo de trocas interregionais, de desenvolvimento local e do refinamento de cerâmicas locais.

A pré história brasileira nos dois últimos milênios é caracterizada pela co-existência de várias culturas na mesma região e é difícil saber o nível de integração entre as diferentes tribos. Não existem evidências de organizações urbanas, a população vivia em sistema tribal, existindo apenas alguma interação econômica entre os diferentes povos ${ }^{1}$.

Existem poucas evidências de migrações periódicas, com exceção de algumas, entre a praia e o interior, no sudeste do Brasil.

Algumas tribos, principalmente as que se dedicavam à agricultura nas planícies aluviais, competiam entre si, mas outros grupos viviam isoladamente e provavelmente em paz, nas regiões entre os $\operatorname{rios}^{2}$.

O material cerâmico, escolhido para o presente estudo, é proveniente dos sítios Guará 1 e Guará 2 localizados no município de Guaráíta, a 185 km de Goiânia, no limite noroeste da micro-região do "Mato Grosso de Goiás", nas coordenadas 50 1' 37" longitude oeste de Greenwich e $15^{\circ} 35^{\prime}$ '58” latitude sul. Os sítios Guará 1 e 2 correspondem a duas ocupações de agricultores pré-coloniais distintos, sendo que o material cerâmico sobre a vertente Nordeste e Este pode ser identificado com a tradição Uru, enquanto os vestígios arqueológicos ao sul, sobre a parte mais elevada da colina, se filiam à

\footnotetext{
*e-mail: sabinoc@cdtn.br
}

tradição Aratu (fase Mossâmedes). Apenas em uma estreita faixa ocorre uma discreta sobreposição de ambas as ocupações, como mostra a Figura $1^{3}$.

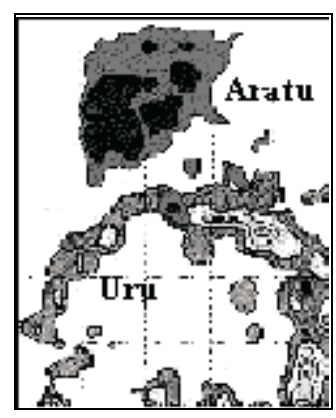

Figura 1. Distribuição das culturas no sítio Guará

O estudo teve como objetivo comparar cerâmicas encontradas nos dois sítios, bem como as características das cerâmicas de cada sítio.

A técnica utilizada foi ativação neutrônica paramétrica $\mathrm{k}_{0}$, no reator nuclear TRIGA IPR R1, localizado no Centro de Desenvolvimento da Tecnologia Nuclear/Comissão Nacional de Energia Nuclear em Belo Horizonte ${ }^{4}$. Para a análise dos dados foram utilizadas as técnicas de componentes principais e ANOVA.

\section{METODOLOGIA}

Escolha das amostras

Os procedimentos amostrais recomendados por Shennan ${ }^{5}$ foram utilizados na escolha das 165 amostras estudadas. A distribuição está apresentada na Tabela 1 e as formas estudadas da cultura Uru estão na Figura 2. 


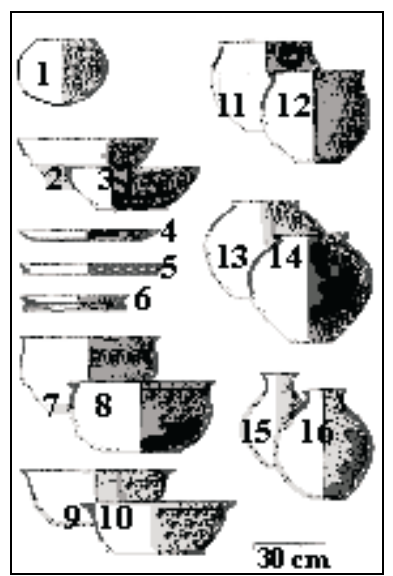

Figura 2. Representação das formas das cerâmicas estudadas da tradição Uru

As formas escolhidas podem ser atribuídas às funções essencialmente distintas, favorecendo uma comparação entre função e qualidade da matéria prima utilizada. No estabelecimento da função dos vasilhames foram levados em consideração não apenas as suas características morfológicas e analogias gerais mas, sobretudo, as marcas de uso tais como presença de fuligem, desgastes por líquidos e atritos mecânicos. Assim, a forma 1 é essencialmente destinada à atividade de cozinhar, a forma 2 a servir e estocar alimentos, as formas 4 e 5 a tostar farinha e assar beijú, a forma 8 ao processamento de alimentos, incluindo a ralagem da mandioca enquanto a forma 16 parece ter sido empregada predominantemente no transporte e na estocagem de líquidos, sobretudo água ${ }^{6}$.

Escolheu-se adicionalmente uma forma da tradição Aratu (forma 20, grande recipiente periforme de contorno infletido).

Seguindo o procedimento amostral, a partir da listagem das formas acima indicadas, escolheram-se, a partir da tabela de números aleatórias, as peças para a análise. Em caso de uma peça escolhida ter tamanho insuficiente, foi selecionada a peça imediatamente posterior ${ }^{5}$.

\section{Procedimento experimental}

Os dados relativos às características físicas, estilísticas e arqueológicas de cada amostra foram anotados. As amostras foram preparadas utilizando-se uma furadeira e brocas de tungstênio. Primeiro foi feita uma limpeza no sentido paralelo, com o objetivo de retirar impurezas depositadas ao longo dos anos. Em seguida as amostras para análise foram retiradas, utilizando-se a broca no sentido transversal. A amostragem foi feita em várias perfurações na superfície previamente limpa. O pó foi coletado em papel especial acondicionado em placa de Petri.

Foram pesadas três alíquotas de $300 \mathrm{mg}$ em tubos de polietileno: uma foi utilizada para determinação de elementos que dão origem a radioisótopos de meia vida curta $(\mathrm{Al}, \mathrm{Cu}, \mathrm{Cl}, \mathrm{Ti}$ e $\mathrm{V})$; outra para meia vida média (As, Au, Ga, K, La e Na) e a terceira para meia vida longa (Ce, Co, Cr, Cs, Fe, Rb, Sc, Ta e Zn).

As diferentes argilas foram aquecidas a $65^{\circ} \mathrm{C}$ durante a noite, sendo em seguida pulverizadas em moinho de ágata. As fontes de argilas eram as mesmas que até pouco tempo foram utilizadas pelos artesãos tradicionais, por serem as existentes na região.

A técnica $\mathrm{k}_{0}$ de ativação neutrônica foi utilizada na determinação da concentração dos elementos, no reator TRIGA IPR R1, cujo fluxo térmico é $6,6 \times 10^{11}$ neutron $\mathrm{cm}^{2} \mathrm{~s}^{-1}$. Para os nuclídeos de meia vida curta foram utilizados 5 min de irradiação, 10 min de espera e 600 s de contagem; para os de meia vida média, 4 h de irradiação, 12 $\mathrm{h}$ de espera e $3 \mathrm{~h}$ de contagem e para os de meia vida longa, $20 \mathrm{~h}$ de irradiação, 15 d de espera e $4 \mathrm{~h}$ de contagem. Para cada 8 amostras uma foi feita em duplicata, com o objetivo de testar a reprodutibilidade.

A espectrometria gama foi feita utilizando um detetor HPGe, CANBERRA, modelo GC1518 com resolução de $1,85 \mathrm{keV}$ para a energia de $1332 \mathrm{keV}$ do ${ }^{60} \mathrm{Co}$. $\mathrm{O}$ detetor foi acoplado ao programa "Maestro", EG \&G Ortec.

Vários materiais de referência foram analisados junto com as amostras, com o objetivo de testar a exatidão : IAEA/Soil-7, IAEA/ Soil-5, GXR-1, GXR-3, GXR-6 (United States Geological Survey).

Todos os dados foram analisados utilizando o SYSTAT 7.0. Nesta análise a distribuição da concentração dos dados foi primeiro transformada em distribuição logaritmo natural. Os componentes principais foram calculados a partir da matriz de covariância e então efetuada ANOVA e t-test, quando necessário 7 .

\section{Análise de dados}

As 20 variáveis químicas estudadas foram resumidas em 5 componentes, explicando $72 \%$ da variabilidade total dos dados. O primeiro fator pode ser explicado como sendo uma comparação entre $\mathrm{Al}$ (negativo) e as demais variáveis (positivas). O primeiro fator indica também uma elevada contribuição positiva $(>0,7)$ do $\mathrm{Co}, \mathrm{Cr}, \mathrm{Sc}$

Tabela 1. Distribuição das amostras de acordo com a tradição, forma e tipo de argila

\begin{tabular}{|c|c|c|c|c|}
\hline Forma & Tradição & Tipo de Argila* & Total global & Número de amostras estudadas \\
\hline Forma \#10 & Uru & 1 & 154 & 15 \\
\hline Forma \# 1 & Uru & 1 & 34 & 15 \\
\hline Forma \# 3 & Uru & 1 & 103 & 15 \\
\hline Forma \# 8 & Uru & 1 & 363 & 15 \\
\hline Forma \# 8 & Uru & 2 & 65 & 15 \\
\hline Forma \# 8 & Uru & 3 & 16 & 15 \\
\hline Forma \# 8 & Uru & 4 & 19 & 15 \\
\hline Forma \# 4 and \# 5 & Uru & 1 & 96 & 15 \\
\hline Forma \#16 & Uru & 1 & 205 & 15 \\
\hline Periforme & Aratu (Mossâmedes) & 5 & 40 & 15 \\
\hline Periforme & Aratu (Mossâmedes) & 3 & 23 & 15 \\
\hline Total & & & 1118 & 165 \\
\hline
\end{tabular}

* 1 - esbranquiçada 2 - amarelada 3 - cor de tijolo 4 - não definida 5 - marrom 
e V. Estes elementos estão correlacionados, apresentando valores maiores que 0,6 na matriz de correlação de Pearson.

Esta correlação está apresentada na Figura 3. O fator 1 (F1) está relacionado principalmente à argila utilizada na confecção da cerâmica, conforme Tabela 1.

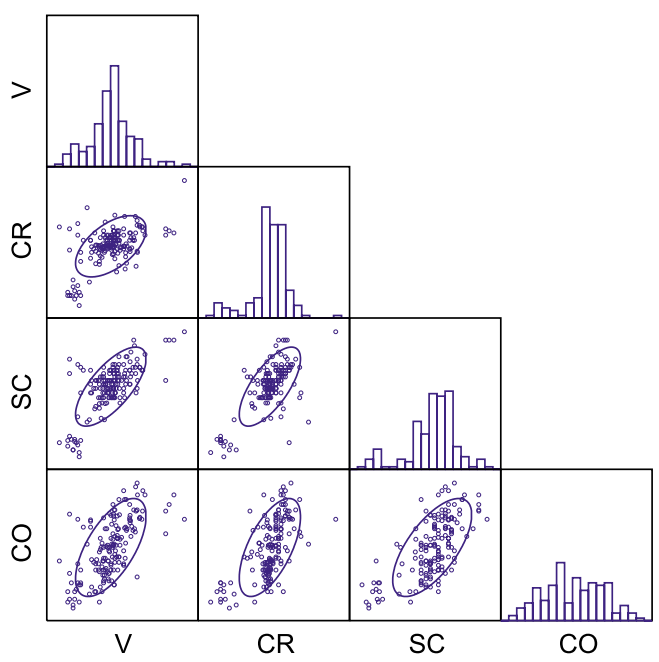

Figura 3. Correlação entre alguns dos elementos relacionados ao F1

O teste-t aplicado às diferentes variáveis relacionadas às características das cerâmicas e ao fator 1 indicou que existe uma diferença significativa (p igual a 0,005) entre as tradições Uru e Aratu. A Figura 4 demostra haver uma nítida diferença entre as duas tradições no referente ao fator $1(\mathrm{p}=0,001)$. As diferentes argilas foram também estudadas em relação a este fator. A probabilidade $\mathrm{p}=0,0000$ indica que estas são também diferentes em relação ao fator. O estudo está apresentado na Figura 5, na qual a numeração das argilas (NTA) é mesma que na Tabela 1.

\section{Least Squares Means}

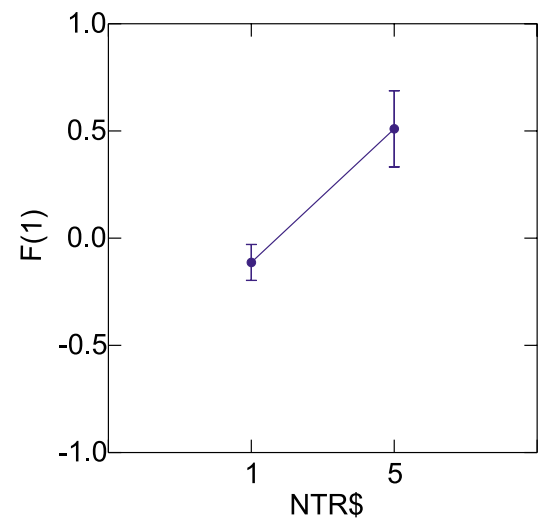

Figura 4. ANOVA aplicada ao F1 e tradições. F1 - Primeiro componente principal, NTR - Tradição : 1 - Uru 5 - Aratu

A mesma diferença é encontrada quando se estuda forma e volume, para a forma $\mathrm{p}=0,0000$ e para o volume $\mathrm{p}=0,012$. No caso de volume os índices indicam : 1 - entre 0 e $1 \mathrm{~L} ; 2$ - entre 1 e $2 \mathrm{~L} ; 3$ entre 2 e $5 \mathrm{~L} ; 4$ - entre 5 e $10 \mathrm{~L} ; 5$ - entre 10 e $20 \mathrm{~L} ; 6$ - entre 20 e 50 L e 7 - maior que 50 L. Os resultados estão apresentados nas Figuras 6 e 7.

\section{Least Squares Means}

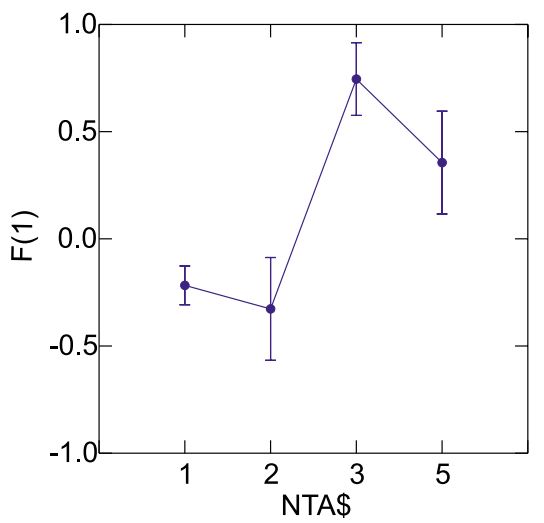

Figura 5. ANOVA aplicada ao F1 e diferentes argilas. F1 - Primeiro componente principal

\section{Least Squares Means}

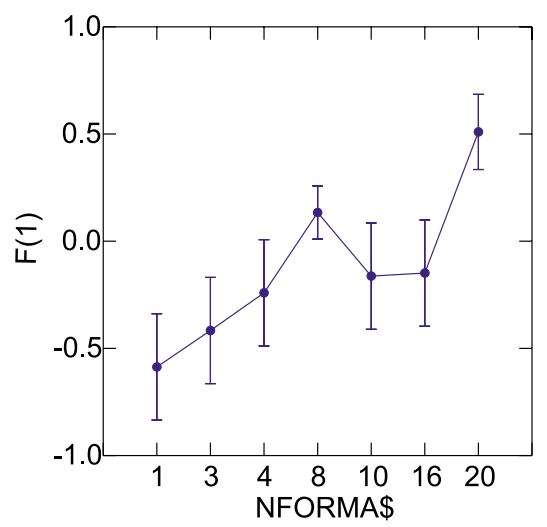

Figura 6. ANOVA aplicada ao F1 e diferentes formas. F1 - Primeiro componente principal, NFORMA\$ corresponde as formas apresentadas na Figura 2

O fator 2 (F2) é composto por acentuada contribuição negativa $(<-0,6)$ do $\mathrm{Cs}$, Rb e Ta e acentuada contribuição positiva $(>0,6)$ do Fe e Na. Esta correlação está apresentada na Figura 8 e o fator 2 está relacionado principalmente ao antiplástico adicionado à argila na confecção das cerâmicas.

\section{Least Squares Means}

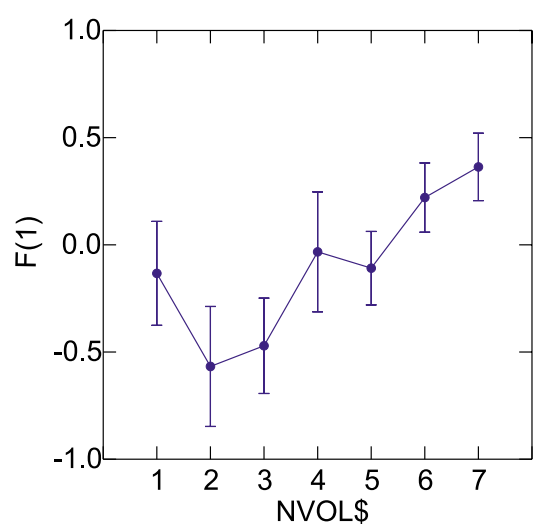

Figura 7. ANOVA aplicada ao F1 e diferentes volumes. F1 - Primeiro componente principal, NVOL $\$$ corresponde aos volumes descritos acima 


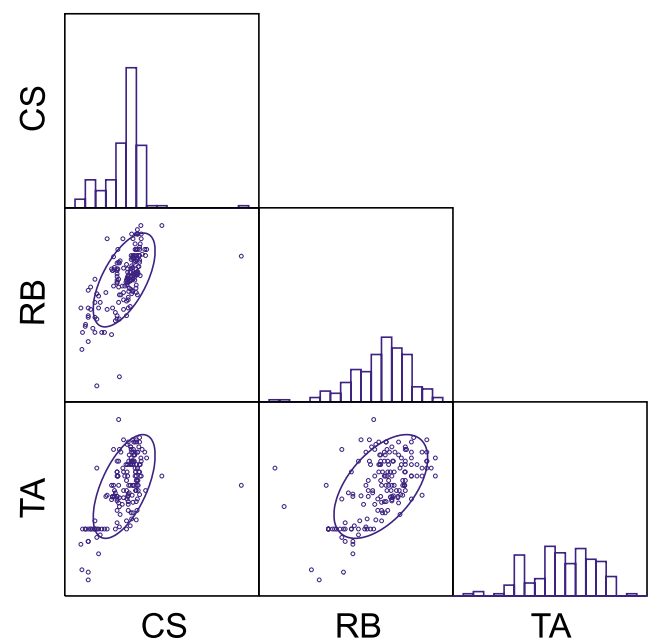

Figura 8. Correlação entre alguns elementos relacionados ao fator 2

As duas tradições não apresentam diferença significativa com relação a este fator $(\mathrm{p}=0,72)$. A característica forma apresenta diferença significativa considerando o fator $2(\mathrm{p}=0.07)$. O mesmo ocorre com o volume $(\mathrm{p}=0,012)$. Os resultados estão apresentados nas Figuras 9 e 10 .

\section{Least Squares Means}

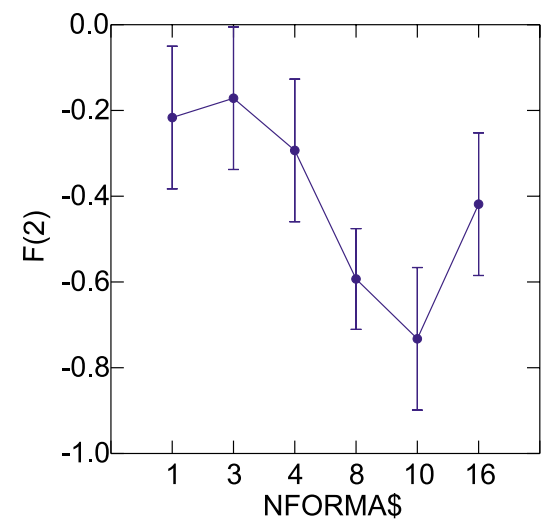

Figura 9. ANOVA aplicada ao F2 e diferentes formas. F2 - Segundo componente principal, NFORMA\$ corresponde as formas apresentadas na Figura 2

$\mathrm{O}$ fator $3(\mathrm{~F} 3)$ se relaciona principalmente às concentrações de $\mathrm{Na}$ e K, mas os resultados são semelhantes aos encontrados com o fator 2. Os demais fatores não levaram a resultados significativos.

\section{CONCLUSÕES}

O método de análise por ativação neutrônica pode ser utilizado no estudo de cerâmicas de tribos brasileiras. O método $\mathrm{k}_{0}$ é especial-

\section{Least Squares Means}

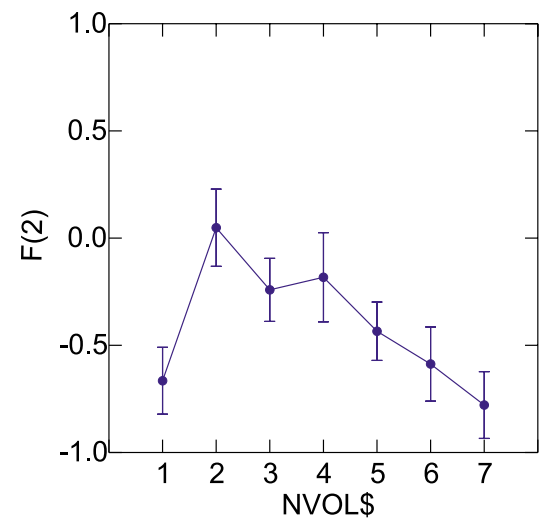

Figura 10. ANOVA aplicada ao F2 e diferentes volumes. F2 - Segundo componente principal, NVOL $\$$ corresponde aos volumes descritos acima

mente indicado por possibilitar a determinação de qualquer elemento detetado na amostra, sem que haja necessidade da utilização de uma curva de padrões.

É possível concluir que as tradições utilizavam fontes de argila diferentes e que também usavam diferentes têmperas.

Tribos simples, como eram a Uru e Aratu, já escolhiam diferentes argilas para confeccionar vasos de diferentes utilidades. Os utensílios utilizados para cozinhar ou ir ao forno apresentam composição diferente dos que eram usados apenas para processar alimentos.

Da mesma maneira é distinta a composição de vasilhames de diferentes volumes, o que indica algum desenvolvimento na técnica de confecção de cerâmicas, através da habilidade e racionalidade tecnológica.

A utilização do mesmo antiplástico pelas duas culturas provavelmente é devida a presença de partículas de cinza vegetal (denominado cariapé A), presente na maioria das peças. Este era um dos principais antiplásticos disponíveis na região.

\section{AGRADECIMENTOS}

Este projeto foi financiado pela Agência Internacional de Energia Atômica e teve o apoio da Comissão Nacional de Energia Nuclear e do Centro de Desenvolvimento da Tecnologia Nuclear.

\section{REFERÊNCIAS}

1. Prous, A.; Brito, M. E; Lima, M.; Revista do Museu de Arqueologia e Etnologia 1994, 4, 71.

2. Prous, A.; Journal de la Société des Américanistes 1996, 77, 77.

3. Wust, I. Dissertação de Mestrado, Universidade de São Paulo, Brasil, 1983.

4. Sabino, C.; Prous, A.; Wust, I.; Amaral, A.; Proceedings of the $1^{\text {st }}$ International Symposium on Nuclear and Related Techniques in Agriculture, Industry, Health and Environment, Cuba, 1997.

5. Shennan, S.; Quantifying Archaeology, Edingburgh University Press, Academic Press, Inc.: New York, 1988.

6. Wust, I.; Carvalho, H.B.; Revista do Museu de Arqueologia e Etnologia 1996, 6, 47 . 